\title{
Innovación docente en Rehabilitación Médica. Cómo conseguir que los alumnos sean el centro de su aprendizaje
}

\section{Teaching innovation in Medical Rehabilitation. How to make students the center of your learning}

AguAS-SANTAS JimÉnEz SARMIENTO.

Orcid: ఏiménez-Sarmiento, AS (https://

orcid.org/0000-0001-6683-8066)

Universidad de Sevilla

Departamento de Farmacología,

Pediatría y Radiología

mjsarmiento@us.es

DOI: http://dx.doi.org/10.12795/9788447231003.034

Pp.: 714-734 


\section{Descripción del contexto.}

La asignatura para la que se pretendía desarrollar el Ciclo de Mejora en el Aula (CIMA) es Rehabilitación Médica. Es una asignatura que se imparte en el segundo cuatrimestre del 4o curso del grado de Medicina. Debido a las fechas en las que se desarrolla el Curso General de Docencia Universitaria, decidí aplicarlo en otra asignatura. Se trata de la asignatura de Patología Médica del grado de Biomedicina. La docencia se realiza a través de seminarios-taller que pretenden acercar al alumno a situaciones clínicas reales, vividas en el ámbito de una consulta médica o planta de hospitalización. Dada la situación de Pandemia por COVID19, se decide que estas sesiones se realicen de forma presencial en grupos de 6 alumnos. Es con uno de estos grupos con los que apliqué el CIMA.

Ciclos de Mejora en el Aula (2020). Experiencias de Innovación Docente de la US Esta obra se distribuye con la licencia Creative Commons 


\section{Diseño del CIMA.}

\section{Mapa de contenidos y problemas.}

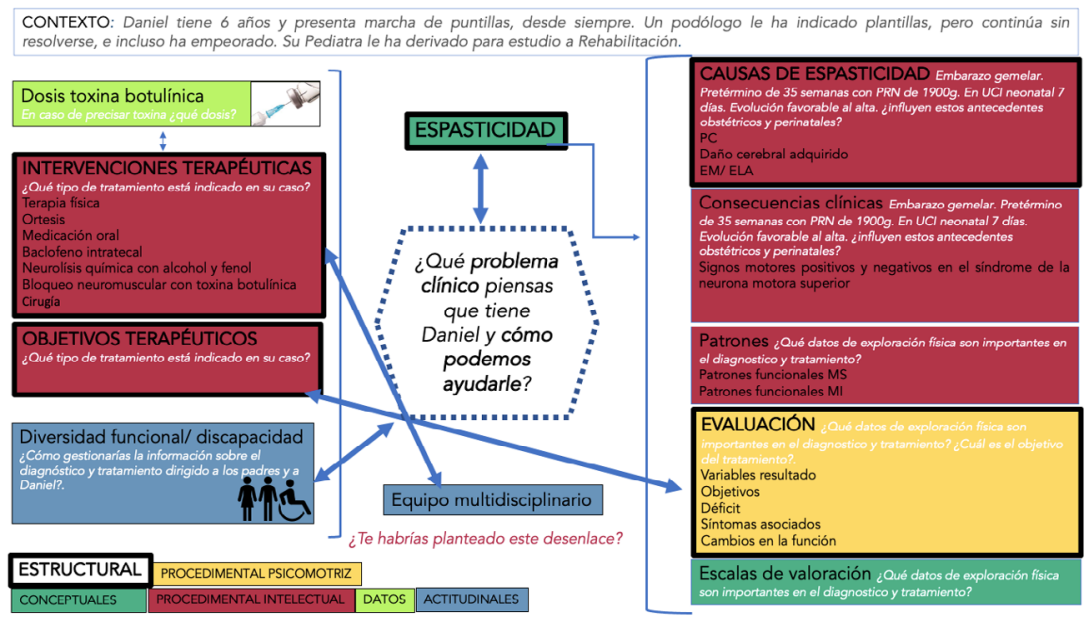

Figura 1. Mapa de contenidos y problemas

Los contenidos de este CIMA se distribuyen en contenidos estructurales y no estructurales, conceptuales, datos, procedimentales (psicomotriz e intelectual) y aquellos que promueven la actitud del alumno (Porlán, 2017).

El CIMA desarrolla el tema de la espasticidad mediante una pregunta o problema clínico que se contextualiza del siguiente modo: "Daniel tiene 6 años y presenta marcha de puntillas. Un Podólogo le ha indicado plantillas, pero su problema continúa sin resolverse e incluso ha empeorado. Su Pediatra le ha derivado para estudio a Rehabilitación". A partir de ahí surge la pregunta: ¿Qué problema clínico piensas que tiene Daniel y cómo podemos ayudarle?

Mediante una secuencia de 6 preguntas -que- son las mismas del cuestionario de ideas iniciales y con el que posteriormente evaluaremos la enseñanza, se va haciendo

Ciclos de Mejora en el Aula (2020). Experiencias de Innovación Docente de la US Esta obra se distribuye con la licencia Creative Commons 
un recorrido por las causas de la espasticidad, las consecuencias clínicas, los patrones espásticos y cómo evaluar la espasticidad, en íntima relación con los objetivos terapéuticos, escalas de valoración, tipos de intervenciones terapéuticas, aportándose datos como las dosis de toxina botulínica necesarias para realizar bloqueos neuromusculares. Finalmente, se abordan aspectos actitudinales como, el enfoque multidisciplinar del problema, aspectos como la diversidad funcional, discapacidad e inclusión.

El CIMA completo tiene 8 horas que se desarrollan en 2 sesiones de 4 .

\section{Modelo metodológico posible.}

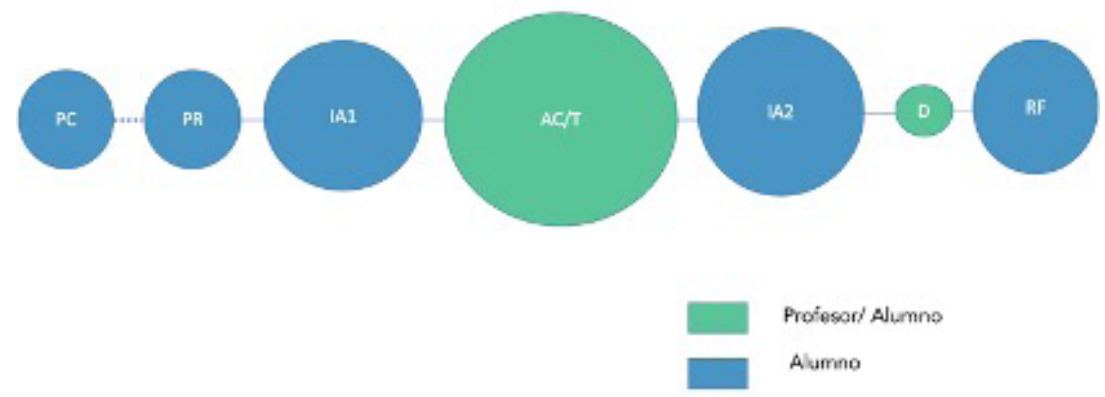

Figura 2. Modelo metodológico posible

Los colores indican la participación principal, verde (profesor y alumnos) y azul (alumnos). PC: Pre-clase. PR: Problema. IA1: Ideas iniciales de los alumnos. AC/T: Actividades de contraste/ Teoría. IA2: Ideas tras el AC/T. D: Dudas de los alumnos. RF: Reflexiones finales.

Este modelo metodológico posible difiere en gran medida del clásico modelo de docencia que se imparte en la asignatura, que se desarrolla mediante clases magistrales. En este nuevo modelo, el mayor peso de las sesiones recae sobre los propios alumnos, los cuales, a través

Ciclos de Mejora en el Aula (2020). Experiencias de Innovación Docente de la US Esta obra se distribuye con la licencia Creative Commons 
de una secuencia de preguntas clínicas y con la imprescindible guía del profesor, van adquiriendo los conocimientos de modo natural, sin artificios y desarrollando la memoria basada en la experiencia, en contraposición a la habitualmente utilizada, la memoria académica (Finkel, 2008).

Para ello, antes de las sesiones, se contacta con los alumnos mediante correo electrónico y se le aporta un enlace a un cuestionario, desarrollado en Google forms con 6 preguntas y un contexto clínico. Se les solicita que lo contesten y envíen de forma anónima, mediante pseudónimo. Además, se les aporta una Guía de Práctica Clínica de espasticidad (Sociedad Española de Rehabilitación y Medicina Física, 2010), con la que vamos a trabajar durante las sesiones.

\section{Secuencia de actividades programadas}

Tabla 1. Secuencia de actividades programadas

\begin{tabular}{|l|l|l|l|}
\hline Actividad & $\begin{array}{l}\text { Leyenda en } \\
\text { el modelo } \\
\text { metodológico }\end{array}$ & Desarrollo & Tiempo \\
\hline 1. *Pre-Clase & PC & $\begin{array}{l}\text { Cuestionario de } \\
\text { ideas iniciales } \\
\text { de los alumnos. } \\
\text { Cuestionario } \\
\text { de expectativas } \\
\text { previas. Guía de } \\
\text { Practica Clínica } \\
\text { Espasticidad. }\end{array}$ & $40^{-}$ \\
\hline $\begin{array}{l}\text { 2. *Planteamiento } \\
\text { del Problema. }\end{array}$ & PR & $\begin{array}{l}\text { Cortometraje } \\
\text { "Cuerdas". } \\
\text { Contextualizar } \\
\text { problema. }\end{array}$ & $40^{-}$ \\
\hline
\end{tabular}

Ciclos de Mejora en el Aula (2020). Experiencias de Innovación Docente de la US Esta obra se distribuye con la licencia Creative Commons 


\begin{tabular}{|l|l|l|l|}
\hline $\begin{array}{l}\text { 3. *Ideas } \\
\text { alumnos 1 } \\
\text { (previas) }\end{array}$ & IA1 & $\begin{array}{l}\text { Respuestas del } \\
\text { cuestionario de } \\
\text { ideas iniciales de } \\
\text { los alumnos. }\end{array}$ & $60^{\prime}$ \\
\hline $\begin{array}{l}\text { 4. *Actividades } \\
\text { de contraste/ } \\
\text { Teoría. }\end{array}$ & AC/T & $\begin{array}{l}\text { Contenidos } \\
\text { representados } \\
\text { en el mapa } \\
\text { de contenidos } \\
\text { y problemas. } \\
\text { Cortometraje }\end{array}$ & $120^{\prime}$ \\
\hline $\begin{array}{l}\text { Fundación lan. } \\
\text { alumnos 2 (tras } \\
\text { el contraste } \\
\text { teórico) }\end{array}$ & IA2 & $\begin{array}{l}\text { Secuencia de } \\
\text { preguntas de } \\
\text { cuestionario de } \\
\text { ideas iniciales. } \\
\text { Role Play. }\end{array}$ & $90^{\prime}$ \\
\hline $\begin{array}{l}\text { 6. *Dudas de los } \\
\text { alumnos. }\end{array}$ & D & $\begin{array}{l}\text { Resolución de } \\
\text { dudas. }\end{array}$ & $30^{\prime}$ \\
\hline $\begin{array}{l}\text { 7. *Reflexiones } \\
\text { finales. }\end{array}$ & RF & $\begin{array}{l}\text { Los alumnos } \\
\text { formulan las } \\
\text { conclusiones } \\
\text { de las sesiones. } \\
\text { Cuestionario } \\
\text { de satisfacción } \\
\text { del alumno. } \\
\text { Feedback con los } \\
\text { alumnos. }\end{array}$ & $60^{\prime}$ \\
\hline
\end{tabular}

1. Pre-clase: Se contacta con los alumnos mediante correo electrónico y se les hace llegar el cuestionario de ideas iniciales del alumno, cuestionario de expectativas previas del alumno y una guía de práctica clínica sobre espasticidad.

Se les solicita a los alumnos que contesten los cuestionarios de forma anónima, mediante pseudónimo.

A) Cuestionario ideas iniciales del alumno:

Contexto: Daniel tiene 6 años y presenta marcha de puntillas, desde siempre. Un Podólogo le ha indicado

Ciclos de Mejora en el Aula (2020). Experiencias de Innovación Docente de la US Esta obra se distribuye con la licencia Creative Commons 
plantillas. Pese a ello, su problema continúa sin resolverse e incluso ha empeorado. Su Pediatra le ha derivado para estudio a Rehabilitación. Antecedentes personales obstétricos, perinatales y postnatales: Embarazo gemelar. Primer gemelo. Parto pretérmino de 35 semanas + 3 días con PRN de 1985g. Estuvo en UCI neonatal 7 días con evolución favorable al alta. Desarrollo psicomotor normal. Antecedentes familiares: Padre con pies planos valgos tratados con bota ortopédica en la infancia. Madre con pies cavos valgos. Exploración física: Funciones superiores conservadas. Marcha de puntillas, no consigue apoyo plantígrado ni marcha de talones. A nivel axial: cabeza y cuello normales. No apreciamos asimetrías en el talle, no dismetría clínica por confrontación directa. Balance articular global libre. Balance muscular: en MMSS 5/5 de forma global, en MMII: psoas, cuádriceps, isquiosurales, extensor primer dedo 5/5, tibial anterior, tibial posterior y tríceps sural 3/5. ROTs rotulianos presentes simétricos, aquileos vivos con clonus bilateral. RCP extensor bilateral. Tono muscular: EMA 0 en flexores de cadera, extensores de rodilla y EMA 2 en triceps sural. Tardieu 2 en tríceps sural. Huella plantar: cavo grado 1, hiperapoyo en cabeza del 5o metatarsiano. Valgo de retropié de 8‥

\section{¿Qué problema clínico piensas que tiene Daniel y cómo podemos ayudarle?}

1. ¿Piensas que influyen estos antecedentes? Arguméntalo.

2. ¿Qué datos de exploración física consideras que son importantes en el diagnóstico y por qué?

3. ¿Qué datos de exploración física consideras que son importantes en el tratamiento y por qué?

4. Si tu fueses su médico ¿Qué tipo de tratamiento indicarías en su caso?

Ciclos de Mejora en el Aula (2020). Experiencias de Innovación Docente de la US Esta obra se distribuye con la licencia Creative Commons 
5. En caso que precise algún tratamiento farmacológico, ¿qué dosis de tratamiento aplicarías?

6. ¿Cómo gestionarías la información sobre el diagnóstico y tratamiento dirigido a los padres y a Daniel.

B) Cuestionario de expectativas previas del alumno:

1. ¿Qué nivel de expectativas tienes respecto a esta clase? Muy alto. Alto. Medio. Bajo.

2. Señala de los siguientes ítems, ¿cuál se adapta mejor a tus expectativas respecto a la clase? Mejora de competencias. Compartir conocimientos. Obtener información. Otras (señalar).

3. Indica tus expectativas previas en relación a la clase.

4. ¿Qué esperas sobre el ambiente de la clase?

5. ¿Qué esperas del profesor/a que impartirá la clase?

2. Planteamiento del problema: La actividad inicia mediante un cortometraje de animación: "Cuerdas". Al finalizar el mismo se realiza un debate mediante Wooclap. Nube de palabras (palabra que describa tu opinión sobre el cortometraje?. Nube de palabras (palabra con la que describirías la personalidad de María). Nube de palabras (palabra con la que describirías al niño de la silla de ruedas). Nube de palabras (palabras con las que relaciones la discapacidad).

Contextualizar el problema:

a) Se presentan imágenes de 3 personas distintas (el niño del corto, el actor español Juan Manuel Montilla "El Langui" afecto de parálisis cerebral (PC) y el actor americano Roy Franck Mitte, conocido por su papel como Walter White Jr en la exitosa serie "Breaking Bad "(2008) de la AMC, donde interpreta a un adolescente con PC, enfermedad que sufre en la vida real. Mediante Wooclap se les pregunta: ¿Qué 
similitudes apreciáis en estas 3 personas/ personajes? ¿Qué diferencias?

b) Se presenta el caso clínico a resolver, que es el caso clínico que han contestado en el cuestionario de ideas iniciales.

3. Ideas de los alumnos 1: Comentamos respuestas facilitadas por los alumnos en el cuestionario de ideas iniciales de la Actividad 1. La profesora lee en voz alta las respuestas que han facilitado los alumnos a cada pregunta, sin hacer ninguna valoración. Acto seguido, se distribuye a los alumnos en grupos de 2 alumnos, se les entrega por escrito el resultado del análisis del cuestionario inicial y se les pide que debatan en grupo, tratando de formular respuestas de grupo.

4. Actividades de contraste/ teoría: Mediante las preguntas encadenadas del cuestionario de ideas iniciales, se tratarán los siguientes contenidos: Espasticidad, causas (se prestará especial atención a la PC como causa. Se retomará la pregunta de los 3 varones afectos de PC para crear conflicto y desarrollar los tipos de PC), consecuencias clínicas, patrones espásticos (se explicarán a través de la APP Localización Muscular para infiltración ecoguiada de Navandú), evaluación, escalas de valoración, otros tipos de evaluación, objetivos terapéuticos, intervenciones terapéuticas, algoritmo de tratamiento espasticidad (Alcobendas, 2015). La actividad se desarrolla mediante actividades de contraste. Esta actividad finaliza abordando la diversidad funcional/ discapacidad mediante un segundo cortometraje de animación, en este caso "Fundación lan" (trata principalmente el concepto de la inclusión). Se realiza wooclap sobre el corto: Nube de palabras (palabra que describa tu opinión sobre el cortometraje). Nube de palabras (palabra con la que describirías al protagonista). Nube de palabras (palabra con la que 
describirías al resto de niños del corto). Nube de palabras (palabras con las que relaciones la inclusión).

5. Ideas de los alumnos 2: Reproducimos caso clínico tras la actividad 4. Dejamos 30 minutos a los alumnos para que reflexionen y replanteen de nuevo el caso clínico en grupos de 2 alumnos. Seguidamente se comentan las respuestas y reflexiones en voz alta.

Se finaliza reformulando respuestas de grupo a todas las preguntas del cuestionario de ideas iniciales. La ultima pregunta del cuestionario: ¿Cómo gestionarías la información sobre el diagnóstico y tratamiento dirigido a los padres y a Daniel?, que responde a contenidos actitudinales, se reformula mediante un role play que representa la escena: en consulta, el médico (1 alumno/a) se dirige a los padres y Daniel (3 alumnos) para informarles sobre el diagnóstico y tratamiento, desde fuera los biomédicos aportarán datos de investigación en parálisis cerebral (2 alumnos). Tras la ejecución del role play, se produce un debate en clase donde los alumnos analizan y opinan sobre el planteamiento realizado.

6. Dudas de los alumnos: Los alumnos plantean sus dudas y las resolvemos de modo reflexivo con respuestas de grupo.

7. Reflexiones finales: To take away. Se responde a la pregunta problema: ¿Qué problema piensas que tiene Daniel y cómo podemos ayudarle? y se elaboran las conclusiones entre todos los alumnos, con la guía de la profesora. Se realiza cuestionario de satisfacción del alumno. El CIMA finaliza mediante un correo electrónico a los alumnos, en el que se recoge un resumen de las principales ideas tratadas durante las sesiones.

Ciclos de Mejora en el Aula (2020). Experiencias de Innovación Docente de la US Esta obra se distribuye con la licencia Creative Commons 


\section{Cuestionario inicial-final}

Decidí utilizar el mismo cuestionario inicial y final, como modo pragmático de valorar el aprendizaje del alumno. A su vez, las preguntas del cuestionario se incluyeron en el mapa de contenidos. Esto se hizo así porque es a través de esta secuencia de preguntas, como se va abordando cada uno de los contenidos que se pretenden desarrollar en las sesiones. Se contextualiza un caso clínico, anteriormente comentado y sobre el mismo una pregunta o problema clínico, que centra las sesiones: ¿qué problema tiene Daniel y cómo ayudarle? El resto de preguntas van dando pie al abordaje de todos los contenidos, de forma ordenada y estructurada.

\section{Aplicación del CIMA}

\section{Resumen de las sesiones}

Las sesiones se desarrollaron de modo fluido, con los alumnos "conectados" todo el tiempo, mostrando interés por aprender y, me atrevo a decir, con entusiasmo por la metodología docente empleada. Los cortometrajes se aceptaron con ilusión y celo. Después del segundo, incluso alguna alumna, claramente emocionada, no pudo contener alguna lágrima. La lectura en voz alta de las principales ideas de los estudiantes en la actividad 1, creó buen ambiente de clase y despertó inquietud, como también el trabajo en equipo y el role play. Se mostraron muy implicados. Me percaté de algún error en la ficha de lectura, que aclaramos y modifiqué sobre la marcha alguna actividad, que consideré innecesaria. Los alumnos resolvieron el caso completamente entre ellos, con mínima ayuda por mi parte. Las sesiones finalizaron con las conclusiones elaboradas por ellos mismos, que salieron de clase muy motivados, contentos y con sensación de haber aprendido mucho más y mejor.

Ciclos de Mejora en el Aula (2020). Experiencias de Innovación Docente de la US Esta obra se distribuye con la licencia Creative Commons 
En el cuestionario de satisfacción contestaron que les había resultado muy interesante el planteamiento de la clase, que no les había dejado indiferente, no eliminarían nada o quizá el wooclap del principio (igual conclusión a la que yo llegué) y como aspecto a mejorar, un alumno sugirió que la parte que se dedica a lectura de un texto, se plantease en grupos y que luego la explicasen entre ellos. Respecto al formador: el $66,7 \%$ le dio un 10 a la profesora y el 33,3\% restante, un 9. El 100\% recomendaría mantener este sistema de aprendizaje.

\section{Evaluación del aprendizaje de los estudiantes}

Los modelos mentales de los estudiantes se representan en una tabla (Porlán, 2017). Se presentan en la tabla 2.

Tabla 2. Modelos mentales de los estudiantes.

¿Qué problema crees que tiene Daniel y cómo podemos ayudarle? Modelo mental 1 (M1), Modelo mental 2 (M2), Modelo mental 3 (M3) y Modelo mental 4 (M4).

\begin{tabular}{|l|l|l|l|l|}
\hline & M1 & M2 & M3 & M4 \\
\hline $\begin{array}{l}\text { 1. Sobre la } \\
\text { influencia de los } \\
\text { antecedentes }\end{array}$ & $\begin{array}{l}\text { Solo considera } \\
\text { los antecedentes } \\
\text { familiares } \\
\text { estructurales del } \\
\text { pie. }\end{array}$ & $\begin{array}{l}\text { Considera } \\
\text { relacionados los } \\
\text { antecedentes } \\
\text { familiares } \\
\text { estructurales } \\
\text { del pie, pero no } \\
\text { descarta que } \\
\text { pueda existir } \\
\text { otra causa. }\end{array}$ & $\begin{array}{l}\text { Establece } \\
\text { relación con los } \\
\text { antecedentes } \\
\text { perinatales } \\
\text { que puedan } \\
\text { condicionar } \\
\text { alteración del } \\
\text { neurodesarrollo. }\end{array}$ & - \\
\hline $\begin{array}{l}\text { 2.Datos } \\
\text { exploratorios } \\
\text { importantes en } \\
\text { el diagnóstico }\end{array}$ & $\begin{array}{l}\text { Solo atiende a } \\
\text { características } \\
\text { exploratorias del } \\
\text { pie). }\end{array}$ & $\begin{array}{l}\text { También tiene } \\
\text { en cuenta el } \\
\text { tono muscular } \\
\text { y los reflejos } \\
\text { osteotendinosos. }\end{array}$ & $\begin{array}{l}\text { Se centra en el } \\
\text { tono muscular, } \\
\text { reflejos } \\
\text { osteotendinosos } \\
\text { y reflejos } \\
\text { patológicos } \\
\text { (clonus). }\end{array}$ & - \\
& \multicolumn{2}{|l}{} & \\
\hline
\end{tabular}

Ciclos de Mejora en el Aula (2020). Experiencias de Innovación Docente de la US Esta obra se distribuye con la licencia Creative Commons 


\begin{tabular}{|c|c|c|c|c|}
\hline $\begin{array}{l}\text { 3.Datos } \\
\text { exploratorios } \\
\text { importantes en } \\
\text { el tratamiento }\end{array}$ & $\begin{array}{l}\text { Solo atiende a } \\
\text { características } \\
\text { exploratorias del } \\
\text { pie y longitud } \\
\text { del tendón de } \\
\text { Aquiles, a fin de } \\
\text { establecer un } \\
\text { tratamiento con } \\
\text { ortesis plantares. }\end{array}$ & $\begin{array}{l}\text { Presta atención } \\
\text { al tono muscular, } \\
\text { pero lo analiza } \\
\text { de modo erróneo } \\
\text { y sin sentido } \\
\text { clínico. }\end{array}$ & $\begin{array}{l}\text { Presta atención } \\
\text { principalmente } \\
\text { al tono muscular } \\
\text { y reflejos } \\
\text { patológicos } \\
\text { (clonus) a fin } \\
\text { de establecer } \\
\text { objetivos } \\
\text { terapéuticos } \\
\text { con fines de } \\
\text { relajación } \\
\text { muscular. }\end{array}$ & - \\
\hline $\begin{array}{l}\text { 4. Sobre el } \\
\text { tratamiento }\end{array}$ & $\begin{array}{l}\text { Se desvía de la } \\
\text { situación clínica } \\
\text { e hipotetiza } \\
\text { diagnóstico de } \\
\text { etiología distinta } \\
\text { a la estructural o } \\
\text { neurológica. }\end{array}$ & $\begin{array}{l}\text { Centra el } \\
\text { tratamiento } \\
\text { en fisioterapia } \\
\text { a fin de tratar } \\
\text { "problemas } \\
\text { musculares" } \\
\text { y ortesis } \\
\text { plantares para } \\
\text { tratar problema } \\
\text { estructural del } \\
\text { pie. }\end{array}$ & $\begin{array}{l}\text { Propone } \\
\text { tratamiento } \\
\text { miorrelajante } \\
\text { pero no } \\
\text { distingue entre } \\
\text { tratamiento } \\
\text { local/general. }\end{array}$ & $\begin{array}{l}\text { Propone } \\
\text { tratamiento } \\
\text { regional con } \\
\text { bloqueo } \\
\text { neuromuscular } \\
\text { con toxina } \\
\text { botulínica. }\end{array}$ \\
\hline $\begin{array}{l}\text { 5.Sobre la dosis } \\
\text { de tratamiento } \\
\text { farmacológico }\end{array}$ & $\begin{array}{l}\text { Plantea que } \\
\text { no precisa } \\
\text { tratamiento } \\
\text { farmacológico. }\end{array}$ & $\begin{array}{l}\text { Plantea dosis } \\
\text { bajas de } \\
\text { tratamiento, } \\
\text { pero no propone } \\
\text { miorrelajante } \\
\text { o se centra en } \\
\text { tratamiento } \\
\text { del dolor (si lo } \\
\text { hubiera). }\end{array}$ & $\begin{array}{l}\text { Plantea dosis } \\
\text { bajas de } \\
\text { benzodiacepinas. }\end{array}$ & $\begin{array}{l}\text { Plantea } \\
\text { tratamiento } \\
\text { local mediante } \\
\text { bloqueo } \\
\text { neuromuscular } \\
\text { con toxina } \\
\text { botulínica. }\end{array}$ \\
\hline $\begin{array}{l}\text { 6. Sobre la } \\
\text { transmisión de } \\
\text { la información } \\
\text { médica al } \\
\text { paciente y } \\
\text { familiares }\end{array}$ & $\begin{array}{l}\text { No contesta } \\
\text { a lo que se le } \\
\text { pregunta. }\end{array}$ & $\begin{array}{l}\text { Se centra en } \\
\text { vocabulario } \\
\text { exento de } \\
\text { tecnicismos y } \\
\text { con información } \\
\text { completa y } \\
\text { rigurosa pero } \\
\text { no contempla } \\
\text { aspectos éticos. }\end{array}$ & $\begin{array}{l}\text { Plantea posibles } \\
\text { conflictos éticos } \\
\text { en la transmisión } \\
\text { de información } \\
\text { clínica sensible, } \\
\text { atendiendo } \\
\text { al código } \\
\text { deontológico } \\
\text { médico que } \\
\text { regula el secreto } \\
\text { profesional. }\end{array}$ & - \\
\hline
\end{tabular}

Ciclos de Mejora en el Aula (2020). Experiencias de Innovación Docente de la US Esta obra se distribuye con la licencia Creative Commons 


\section{Escaleras de aprendizaje}

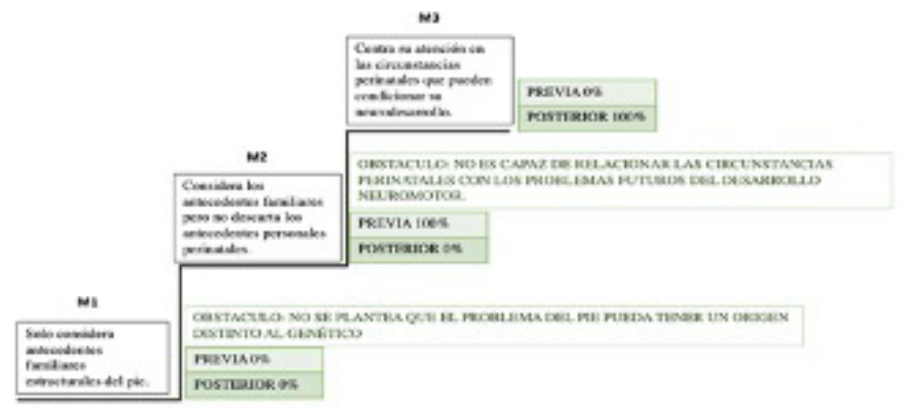

Figura 3. Escalera de la pregunta 1.

Aborda los antecedentes personales del paciente como modo de desarrollar las causas y consecuencias clínicas de la espasticidad. Todos los alumnos se encuentran inicialmente en el nivel M2. Al finalizar las sesiones el 100\% evolucionan al M3, de modo que son capaces de relacionar las circunstancias perinatales con la sintomatología del paciente.

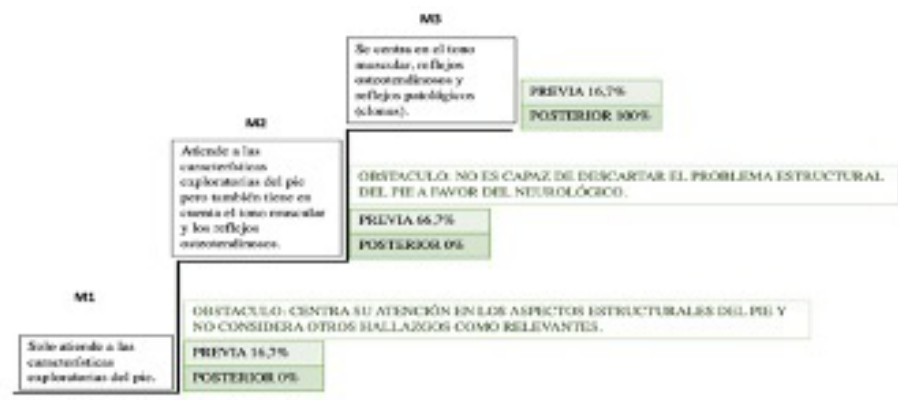

Figura 4. Escalera de la pregunta 2.

Aborda los aspectos de la exploración física que son relevantes para el diagnóstico, centrando el tema del tono muscular, debilidad muscular y alteración de los reflejos osteotendinosos. Inicialmente los alumnos se encontraban entre los niveles M1 y M2 y al finalizar las sesiones, todos ellos evolucionan al nivel M3, de modo que superan el obstáculo de considerar sólo el problema estructural del pie, a favor del problema neurológico.

Ciclos de Mejora en el Aula (2020). Experiencias de Innovación Docente de la US Esta obra se distribuye con la licencia Creative Commons 
Tabla 3. Cuadro de progresión individual del alumno

\begin{tabular}{|c|c|c|c|c|c|c|c|}
\hline $\mathrm{AL} / \mathrm{RESP}$ & 1 & 2 & 3 & 4 & 5 & 6 & EVOLUCION \\
\hline $\mathrm{A} 1$ & $M 2$ & $M 2$ & $M 2$ & $M 2$ & $M 2$ & $M 2$ & +8 \\
\hline $\mathrm{A} 2$ & $M 1$ & $M 2$ & $M 1$ & $M 2$ & $M 2$ & $M 2$ & +10 \\
\hline $\mathrm{A} 3$ & $M 1$ & $M 1$ & $M 1$ & $M 2$ & $M 1$ & $M 2$ & +12 \\
\hline$A 4$ & $M 1$ & $M 2$ & $M 2$ & $M 1$ & $M 2$ & $M 2$ & +10 \\
\hline$A 5$ & $M 2$ & $M 3$ & $M 2$ & $M 3$ & $M 3$ & $M 1$ & +6 \\
\hline$A 6$ & $M 2$ & $M 2$ & $M 2$ & $M 2$ & $M 2$ & $M 1$ & +9 \\
\hline
\end{tabular}

En el cuadro de progresión individual del alumno se puede apreciar, el nivel del que inicialmente partía cada alumno en cada pregunta y el sumatorio final de evolución de todas las preguntas, que se detalla en la ultima columna. El 100\% de los alumnos ha evolucionado durante las sesiones, ninguno de ellos ha involucionado o se ha estacionado y el resultado final es satisfactorio, tanto de forma individual como de forma global.

\section{Evaluación del CIMA.}

\section{Cuestiones a mantener y cambios a introducir para un futuro Ciclo}

Una vez aplicado el CIMA encuentro algunas fortalezas claras que deben centrar el desarrollo de los futuros. Estas son principalmente el haber conseguido a través de una pregunta o problema clínico vehicular el conocimiento. Considero fundamentales el cuestionario de ideas iniciales, leerlo y debatirlo en clase, constituir grupos para re-elaborarlo, antes y después de las actividades de contraste teórico. Así se consigue que el conocimiento fluya de modo natural, sin artificios y sin hacer uso de la memoria académica. Desarrollar la clase a partir del planteamiento

Ciclos de Mejora en el Aula (2020). Experiencias de Innovación Docente de la US Esta obra se distribuye con la licencia Creative Commons 
de un problema es muy útil. Esto en Medicina, se consigue de forma muy concreta a través de los casos clínicos. Estos casos clínicos ponen en contexto al alumno y lo mantienen expectante durante la sesión. Es muy habitual, dentro del modelo metodológico de enseñanza tradicional, mediante clases magistrales, utilizar el caso clínico al final de la clase. Es mucho más interesante presentarlo al principio y valorar las ideas iniciales de las que parten los alumnos, pues de esa manera, se hace un diagnóstico del conocimiento de los alumnos y la enseñanza se orienta a las necesidades que demandan los mismos (Finkel, 2008)

Para un futuro CIMA pretendo introducir más sesiones de trabajo en equipo y reducir el trabajo individual. Considero que al trabajar en grupo, los alumnos desarrollan mejor sus habilidades, sus capacidades y se estimulan entre ellos. Pretendo evitar las diapositivas repletas de información, recurrir a la información completa y actualizada en internet durante las propias clases y plantear al resto de docentes de la asignatura, lo conveniente de modificar los sistemas de evaluación. Porque evaluar no es igual que calificar.

\section{Aspectos de la experiencia que se pretenden incorporar a la práctica}

Los aspectos más útiles que suponen un antes y un después en mi practica docente son los siguientes. En primer lugar, llevar las sesiones preparadas mediante guion y partir de un problema clínico, con una secuencia de preguntas encadenadas, a través de las cuales se van tratando todos los contenidos (Bain, 2005). Hacer un diagnóstico previo de los niveles de conocimiento del que parten los alumnos, mediante un cuestionario de ideas iniciales, sobre el que trabajaremos, principalmente en equipo también durante las sesiones, a través de la realización de 
actividades de contraste, que sirvan como fuente de inspiración orientada. También me resulta de gran utilidad, el cuestionario de expectativas previas del alumno y el de satisfacción posterior. De este modo, como docente valoras las necesidades de los alumnos y los aspectos a mejorar en futuras sesiones.

\section{Principios Didácticos argumentados}

Los principios didácticos empleados y que van a formar parte de mi metodología docente futura son los siguientes:

1. Hacer diagnosis inicial del nivel de conocimiento del que parten los alumnos. Esto se hace mediante cuestionario de ideas iniciales. El sistema tradicional de enseñanza parte del desconocimiento del nivel de formación del alumno sobre el tema a tra$t^{5}$ ar, por lo que no es congruente y no aporta calidad a la enseñanza. Es más útil conocer la situación desde la que se parte para concretar los contenidos a desarrollar.

2. Plantear las sesiones mediante la resolución de un problema (Bain, 2005). En Medicina es muy útil el uso de un caso clínico/ pregunta clínica. Se consigue acercar al alumno a situaciones reales, en las que va a necesitar emplear la lógica. Es un modo de dinamizar la enseñanza y de conseguir la participación activa del alumno.

3. Evitar el uso de diapositivas repletas de información para memorizar. Son contenidos que se memorizan y se olvidan rápido. No quiero que los alumnos soliciten la sesión powerpoint, pues será señal de que pretenden estudiar su contenido. Los contenidos deben haberse tratado en las sesiones y completarse con actividades fuera de las mismas.

4. Recurrir al trabajo fuera del aula para compensar la ausencia de tiempo según el plan docente actual. 
Habría que tener en cuenta que las clases "tipo" en Medicina son de $1 \mathrm{~h}$, en las que se pretende "explicar" grandes volúmenes de información, que se hace tedioso y poco funcional. Habrá que recurrir a trabajo fuera del aula para completar el resto de contenidos que se incluyen en el programa docente y que, por lo tanto, el alumno debe conocer. Lo ideal sería llegar a remodelar el sistema tradicional de clases y aumentar las horas diarias en pro de favorecer el desarrollo de estos nuevos modelos didácticos.

5. Evaluar no es calificar (Porlán, 2017). El sistema tradicional que evalúa mediante examen no es coherente posteriormente con el aprendizaje del alumno, tanto si se trata de un examen de desarrollo (mejoran los resultados aquellos alumnos con buena memoria) como si se trata de un examen tipo test (se ha probado que los alumnos finalmente estudian para saber contestar el test y no para aprender). En Medicina, existe un examen de Médicos Internos Residentes (MIR), de cuyos resultados, sumados al expediente académico, depende la elección de la especialidad y hospital para formarse como especialista. Considero que esto condiciona el aprendizaje, de modo que el alumno estudia para aprobar/ sacar buena calificación y posicionarse en un ranking, lo cual puede crear mal ambiente de estudio, entorno hostil de competencia "no sana". Mediante un sistema de evaluación más global y continuo, se conseguiría estimular el desarrollo de otras competencias, que no se evalúan en examen y que acercan al alumno a una situación más real. No digo que no exista examen, sino que deben existir otros puntos/ aspectos evaluables que tengan igual peso o, incluso más, en la calificación del alumno.

6. Hacer buen uso de las Tecnologías de la Información y Comunicación (TICS). Nos encontramos en la era del conocimiento y de las TICs a "golpe de 
click". En términos generales suman más que restan, pero haciendo uso correcto. Es un modo indudable de acercamiento al alumno, a su generación, pero no debemos caer en el error de utilizarlas de modo indiscriminado y/o siempre las mismas, porque de esa manera dejan de ser útiles y se convierten en nuevos obstáculos de la enseñanza actual.

7. Evaluar la satisfacción de los alumnos a través de cuestionario. Es un modo que el docente tiene para retroalimentarse y poder establecer nuevas líneas estratégicas, eliminar las que no funcionan y mantener las que sí.

Palabras clave: Rehabilitación médica, grado de Medicina, docencia universitaria, experimentación docente universitaria, espasticidad.

Keywords: Medical Rehabilitation, Medical Degree, University Teaching, University Teaching Experimentation, Spasticity.

Ciclos de Mejora en el Aula (2020). Experiencias de Innovación Docente de la US Esta obra se distribuye con la licencia Creative Commons 


\section{Referencias bibliográficas}

Alcobendas-Maestro, M et al. (2015). Guía de Práctica Clínica para el tratamiento de la espasticidad espinal con toxina botulínica. Rehabilitación, 49(1):38-44.

Bain, K. (2005). Lo que hacen los mejores profesores universitarios. Valencia: Publicaciones Universidad de Valencia.

Finkel, D. (2008). Dar clases con la boca cerrada. Valencia: Publicaciones Universidad de Valencia.

Grupo de trabajo de la Sociedad Española de Rehabilitación y Medicina Física (SERMEF) 2010. Guía de práctica clínica para el tratamiento de la espasticidad con toxina botulínica. Sociedad Española de Rehabilitación y Medicina Física.

Porlán, R. (2017). Enseñanza universitaria. Cómo mejorarla. Madrid: Morata.

Ciclos de Mejora en el Aula (2020). Experiencias de Innovación Docente de la US Esta obra se distribuye con la licencia Creative Commons 